\title{
Selection of Polymorphic Structure in Dihydroxystearic Acid/Octyl dihydroxystearate/RBD Palm Kernel Olein \& Medium Chain Triglycerides in Cosmetic and Personal Care Formulation
}

\begin{abstract}
Dihydroxystearic acid (DHSA) and octyl dihydroxystearate (DHSA-octyl ester) have been successfully prepared from palm oleic acid. Preliminary results showed that these compounds are suitable in personal care and cosmetics products. The objective is to study the phase behaviors in ternary system of DHSA/DHSA-octyl ester/RBD palm kernel olein (RBDPKOo) and medium chain triglycerides (MCT) at $85^{\circ} \mathrm{C}$. The phase changes were observed through polarizing light while the formation of texture was confirmed using polarizing microscope combination with heating. From the ternary phase diagram, results showed that all ratios of DHSA/DHSA-octyl ester were completely in two-phase region with various concentrations of RBDPKOo/MCT. Needles and spherulite textures were found in this system. The viscosity and thixotropy of these mixtures were also determined using rheometer. DHSA and DHSAoctyl ester has a melting point of $84.4^{\circ} \mathrm{C}$ and $64.3^{\circ} \mathrm{C}$, respectively.
\end{abstract}

Keyword: Dihydroxystearic acid, octyl dihydroxystearate, RBD palm kernel olein, medium chain triglycerides, ternary phase diagram 